\title{
Univalence Conditions for Two General Integral Operators
}

\author{
Adriana Oprea1, Daniel Breaz ${ }^{2}$ \\ ${ }^{1}$ Department of Mathematics, University of Pitești, Pitești, Romania \\ 2Department of Mathematics, "1 Decembrie 1918" University of Alba lulia, Alba Iulia, Romania \\ Email: adriana oprea@yahoo.com, dbreaz@uab.ro
}

Received 5 June 2014; revised 1 July 2014; accepted 10 July 2014

Copyright (C) 2014 by authors and Scientific Research Publishing Inc.

This work is licensed under the Creative Commons Attribution International License (CC BY).

http://creativecommons.org/licenses/by/4.0/

c) (i) Open Access

\section{Abstract}

Let $A$ be the class of all analytic functions which are analytic in the open unit disc $U=\{z:|z|<1\}$. In this paper we study the problem of univalence for the following general integral operators:

$$
\begin{aligned}
& F_{n}(z)=\int_{0}^{z} \prod_{i=1}^{n}\left(\frac{f_{i}(t)}{t} e^{g_{i}(t)}\right)^{\alpha_{i}} \mathrm{~d} t, \\
& G_{n}(z)=\int_{0}^{z} \prod_{i=1}^{n}\left(f_{i}^{\prime}(t) \mathrm{e}^{g_{i}(t)}\right)^{\beta_{i}} \mathrm{~d} t,
\end{aligned}
$$

in the open unit disc $U$, when $f_{i}, g_{i} \in A, \alpha_{i}, \beta_{i} \in C$.

\section{Keywords}

Analytic Functions, Integral Operators, General Schwarz Lemma

\section{Introduction}

Let $U=\{z:|z|<1\}$ be the unit disk and $A$ be the class of all functions of the form

$$
f(z)=z+\sum_{k=2}^{\infty} a_{k} z^{k}, \quad z \in U
$$

which are analytic in $U$ and satisfy the conditions

$$
f(0)=f^{\prime}(0)-1=0 .
$$


We denote by $S$ the class of univalent and regular functions.

In order to derive our main results, we have to recall here the following univalence conditions.

Theorem 1.1. [1] (Becker's univalence criterion).

If the function $f$ is regular in unit disk $U, f(z)=z+a_{2} z^{2}+\cdots$ and

$$
\left(1-|z|^{2}\right)\left|\frac{z f^{\prime \prime}(z)}{f^{\prime}(z)}\right| \leq 1 \text {, for all } z \in U \text {, }
$$

then the function $f$ is univalent in $U$.

Theorem 1.2. [2] If the function $g$ is regular in $U$ and $|g(z)|<1$ in $U$, then for all $\xi \in U$ the following inequalities hold

$$
\left|\frac{g(\xi)-g(z)}{1-\overline{g(z)} g(\xi)}\right| \leq\left|\frac{\xi-z}{1-\bar{z} \xi}\right|
$$

and

$$
\left|g^{\prime}(z)\right| \leq \frac{1-|g(z)|^{2}}{1-|z|^{2}} .
$$

the equalities hold in case $g(z)=\varepsilon \frac{z+u}{1+\bar{u} z}$ where $|\varepsilon|=1$ and $|u|<1$.

Remark 1.3. [2] For $z=0$, from inequality (3) we obtain for every $\xi \in U$

$$
\left|\frac{g(\xi)-g(0)}{1-\overline{g(0)} g(\xi)}\right| \leq|\xi|
$$

and, hence

$$
|g(\xi)| \leq \frac{|\xi|+|g(0)|}{1+|g(0)||\xi|} .
$$

Considering $g(0)=a$ and $\xi=z$, then

$$
|g(z)| \leq \frac{|z|+|a|}{1+|a||z|}
$$

for all $z \in U$.

\section{Main Results}

In this paper we study the univalence of the following general integral operators:

$$
F_{n}(z)=\int_{0}^{z} \prod_{i=1}^{n}\left(\frac{f_{i}(t)}{t} \mathrm{e}^{g_{i}(t)}\right)^{\alpha_{i}} \mathrm{~d} t,
$$

where $f_{i}, g_{i} \in A$ and $\alpha_{i} \in C$,

$$
G_{n}(z)=\int_{0}^{z} \prod_{i=1}^{n}\left(f_{i}^{\prime}(t) \mathrm{e}^{g_{i}(t)}\right)^{\beta_{i}} \mathrm{~d} t
$$

where $f_{i}, g_{i} \in A$ and $\beta_{i} \in C$.

Theorem 2.1. Let $\alpha_{n} \in C, \quad f_{n} \in S, \quad f_{n}(z)=z+a_{2}^{n} z^{2}+\cdots, \quad n \in N^{*}, \quad g_{n} \in S, \quad g_{n}(z)=z+b_{2}^{n} z^{2}+\cdots$, $n \in N^{*}$,

If

$$
\left|\frac{z f_{n}^{\prime}(z)-f_{n}(z)}{z f_{n}(z)}\right| \leq 1
$$


for all $n \in N^{*}$, for all $z \in U$ and

$$
\begin{gathered}
\left|g_{n}^{\prime}(z)\right| \leq 1 \\
\frac{\left|\alpha_{1}\right|+\left|\alpha_{2}\right|+\cdots+\left|\alpha_{n}\right|}{\left|\alpha_{1} \alpha_{2} \cdots \alpha_{n}\right|}<1, \\
\left|\alpha_{1} \alpha_{2} \cdots \alpha_{n}\right| \leq \frac{1}{\max _{|z| \leq 1}\left[2\left(1-|z|^{2}\right)|z| \frac{|z|+|c|}{1+|c||z|}\right]} .
\end{gathered}
$$

where

$$
|c|=\frac{\left|\alpha_{1}\left(a_{2}^{1}+1\right)+\cdots+\alpha_{n}\left(a_{2}^{n}+1\right)\right|}{2\left|\alpha_{1} \alpha_{2} \cdots \alpha_{n}\right|}
$$

then the function

$$
F_{n}(z)=\int_{0}^{z} \prod_{i=1}^{n}\left(\frac{f_{i}(t)}{t} \mathrm{e}^{g_{i}(t)}\right)^{\alpha_{i}} \mathrm{~d} t
$$

is in the class $S$.

Proof. We have $f_{n} \in S, \frac{f_{n}(z)}{z} \neq 0$, for all $n \in N^{*}$ and $\left(\frac{f_{1}(z)}{z} \mathrm{e}^{g_{1}(z)}\right)^{\alpha_{1}} \cdots\left(\frac{f_{n}(z)}{z} \mathrm{e}^{g_{n}(z)}\right)^{\alpha_{n}}=1$, when $z=0$.

Let us consider the function:

$$
h(z)=\frac{1}{2\left|\alpha_{1} \alpha_{2} \cdots \alpha_{n}\right|} \frac{F_{n}^{\prime \prime}(z)}{F_{n}^{\prime}(z)} .
$$

From (6), we have:

$$
F_{n}^{\prime}(z)=\prod_{i=1}^{n}\left(\frac{f_{i}(z)}{z} \mathrm{e}^{g_{i}(z)}\right)^{\alpha_{i}}
$$

and

$$
F_{n}^{\prime \prime}(z)=\sum_{i=1}^{n} \alpha_{i}\left(\frac{f_{i}(z)}{z} \mathrm{e}^{g_{i}(z)}\right)^{\alpha_{i}-1}\left(\frac{z f_{i}^{\prime}(z)-f_{i}(z)}{z^{2}} \mathrm{e}^{g_{i}(z)}+\frac{f_{i}(z)}{z} \mathrm{e}^{g_{i}(z)} g_{i}^{\prime}(z)\right) \prod_{\substack{k=1 \\ k \neq i}}^{n}\left(\frac{f_{k}(z)}{z} \mathrm{e}^{g_{k}(z)}\right)^{\alpha_{k}} .
$$

From (13) and (14), we have:

$$
\frac{F_{n}^{\prime \prime}(z)}{F_{n}^{\prime}(z)}=\sum_{i=1}^{n} \alpha_{i}\left(\frac{z f_{i}^{\prime}(z)-f_{i}(z)}{z f_{i}(z)}+g_{i}^{\prime}(z)\right)
$$

Using relations before the function $h$ has the form:

$$
h(z)=\frac{1}{2\left|\alpha_{1} \alpha_{2} \cdots \alpha_{n}\right|} \sum_{i=1}^{n} \alpha_{i}\left(\frac{z f_{i}^{\prime}(z)-f_{i}(z)}{z f_{i}(z)}+g_{i}^{\prime}(z)\right) .
$$

We have:

$$
h(0)=\frac{1}{2\left|\alpha_{1} \alpha_{2} \cdots \alpha_{n}\right|} \alpha_{1}\left(a_{2}^{1}+1\right)+\frac{1}{2\left|\alpha_{1} \alpha_{2} \cdots \alpha_{n}\right|} \alpha_{2}\left(a_{2}^{2}+1\right)+\cdots+\frac{1}{2\left|\alpha_{1} \alpha_{2} \cdots \alpha_{n}\right|} \alpha_{n}\left(a_{2}^{n}+1\right) .
$$

By using the relations (15), (8) and (9), we obtain: 


$$
\begin{gathered}
|h(z)| \leq \frac{1}{2\left|\alpha_{1} \alpha_{2} \cdots \alpha_{n}\right|} \sum_{i=1}^{n}\left|\alpha_{i}\left(\frac{z f_{i}^{\prime}(z)-f_{i}(z)}{z f_{i}(z)}+g_{i}^{\prime}(z)\right)\right| \leq \frac{1}{2\left|\alpha_{1} \alpha_{2} \cdots \alpha_{n}\right|} 2 \sum_{i=1}^{n}\left|\alpha_{i}\right| \leq 1 \\
|h(0)|=\frac{\left|\alpha_{1}\left(a_{2}^{1}+1\right)+\cdots+\alpha_{n}\left(a_{2}^{n}+1\right)\right|}{2\left|\alpha_{1} \alpha_{2} \cdots \alpha_{n}\right|}=|c| .
\end{gathered}
$$

Applying Remark 1.3 for the function $h$, we obtain:

$$
|h(z)|=\frac{1}{2\left|\alpha_{1} \alpha_{2} \cdots \alpha_{n}\right|}\left|\frac{F_{n}^{\prime \prime}(z)}{F_{n}^{\prime}(z)}\right| \leq \frac{|z|+|h(0)|}{1+|h(0)||z|} \leq \frac{|z|+|c|}{1+|c||z|} .
$$

From (18), we get:

$$
\left|\left(1-|z|^{2}\right) z \frac{F_{n}^{\prime \prime}(z)}{F_{n}^{\prime}(z)}\right| \leq\left|\alpha_{1} \alpha_{2} \cdots \alpha_{n}\right| 2\left(1-|z|^{2}\right)|z| \frac{|z|+|c|}{1+|c||z|},
$$

for all $z \in U$.

Let us consider the function: $H:[0,1] \rightarrow R$

$$
H(x)=2\left(1-x^{2}\right) x \frac{x+|c|}{1+|c| x}, x=|z| .
$$

Since $H\left(\frac{1}{2}\right)=\frac{3}{4} \frac{1+2|c|}{2+|c|}>0$, it results:

$$
\max _{x \in[0,1]} H(x)>0 .
$$

Using this result and the form (19), we have:

$$
\left|\left(1-|z|^{2}\right) z \frac{F_{n}^{\prime \prime}(z)}{F_{n}^{\prime}(z)}\right| \leq\left|\prod_{i=1}^{n} \alpha_{i}\right| \max _{|z|<1}\left[2\left(1-|z|^{2}\right)|z| \frac{|z|+|c|}{1+|c||z|}\right],
$$

for all $z \in U$.

Applying the condition (10) in relation (20), we obtain:

$$
\left(1-|z|^{2}\right)\left|\frac{z F_{n}^{\prime \prime}(z)}{F_{n}^{\prime}(z)}\right| \leq 1,
$$

for all $z \in U$ and from Theorem 1.1, we have $F_{n} \in S$.

Corollary 2.2. Let $\alpha$ be a complex number and the functions $f \in S, f(z)=z+a_{2} z^{2}+\cdots, g \in S$, $g(z)=z+b_{2} z^{2}+\cdots$. If

$$
\left|\frac{z f^{\prime}(z)-f(z)}{z f(z)}\right|<1 \text { and }\left|g^{\prime}(z)\right|<1
$$

for all $z \in U$ and the constant $|\alpha|$ satisfies the condition:

$$
|\alpha| \leq \frac{1}{\max _{|z| \leq 1}\left[2|z|\left(1-|z|^{2}\right) \frac{2|z|+\left|a_{2}+1\right|}{2+\left|a_{2}+1\right||z|}\right]},
$$

then the function

$$
F_{1}(z)=\int_{0}^{z}\left(\frac{f(t)}{t} \mathrm{e}^{g(t)}\right)^{\alpha} \mathrm{d} t
$$


is in the class $S$.

Proof. We consider $n=1$ in Theorem 2.1.

Remark 2.3. For $n=1, \mathrm{e}^{g_{1}(t)}=1, \quad \alpha_{1}=1$ and $f_{1}=f$ in relation (11), we obtain the integral operator $I(z)=\int_{0}^{z} \frac{f(t)}{t} \mathrm{~d} t$, introduced by J. W. Alexander in [3].

Remark 2.4. For $n=1, \mathrm{e}^{g_{1}(t)}=1, \alpha_{1}=\alpha, f_{1}=f$ in relation (6), we obtain the integral operator $F(z)=\int_{0}^{z}\left(\frac{f(t)}{t}\right)^{\alpha} \mathrm{d} t$, defined and studied by V. Pescar in [4] [5].

Remark 2.5. For $\mathrm{e}^{g_{i}(t)}=1$, for all $i=1, \cdots, n$, we get the integral operator $I_{n}(z)=\int_{0}^{1} \prod_{i=1}^{n}\left(\frac{f_{i}(t)}{t}\right)^{\alpha_{i}} \mathrm{~d} t$, $z \in U$ studied by D. Breaz, N. Breaz in [6] and D. Breaz in [7].

Theorem 2.6.

Let $\beta_{n} \in C, \quad f_{n} \in S, \quad f_{n}(z)=z+a_{2}^{n} z^{2}+\cdots, \quad n \in N^{*}, \quad g_{n} \in S, \quad g_{n}(z)=z+b_{2}^{n} z^{2}+\cdots, \quad n \in N^{*}$. If

$$
\left|\frac{f_{n}^{\prime \prime}(z)}{f_{n}^{\prime}(z)}\right| \leq 1
$$

for all $n \in N^{*}$, for all $z \in U$ and $\left|g_{n}^{\prime}(z)\right| \leq 1$

$$
\begin{gathered}
\frac{\left|\beta_{1}\right|+\left|\beta_{2}\right|+\cdots+\left|\beta_{n}\right|}{\left|\beta_{1} \beta_{2} \cdots \beta_{n}\right|}<1, \\
\left|\prod_{i=1}^{n} \beta_{i}\right| \leq \frac{1}{\max _{|z| \leq 1}\left[2\left(1-|z|^{2}\right)|z| \frac{|z|+|c|}{1+|c||z|}\right]},
\end{gathered}
$$

where

$$
|c|=\frac{\left|\beta_{1}\left(2 a_{2}^{1}+1\right)+\cdots+\beta_{n}\left(2 a_{2}^{n}+1\right)\right|}{2\left|\beta_{1} \beta_{2} \cdots \beta_{n}\right|}
$$

then the function

$$
G_{n}(z)=\int_{0}^{z} \prod_{i=1}^{n}\left(f_{i}^{\prime}(t) \mathrm{e}^{g_{i}(t)}\right)^{\beta_{i}} \mathrm{~d} t
$$

is in the class $S$.

Proof. We have $f_{n} \in S$, for all $n \in N^{*}$ and $\left(f_{1}^{\prime}(z) \mathrm{e}^{g_{1}(z)}\right)^{\beta_{1}} \cdots\left(f_{n}^{\prime}(z) \mathrm{e}^{g_{n}(z)}\right)^{\beta_{n}}=1$, when $z=0$.

Let us consider the function:

$$
p(z)=\frac{1}{2\left|\beta_{1} \beta_{2} \cdots \beta_{n}\right|} \frac{G_{n}^{\prime \prime}(z)}{G_{n}^{\prime}(z)} .
$$

From (27), we have:

$$
G_{n}^{\prime}(z)=\prod_{i=1}^{n}\left(f_{i}^{\prime}(z) \mathrm{e}^{g_{i}(z)}\right)^{\beta_{i}}
$$

and

$$
G_{n}^{\prime \prime}(z)=\sum_{i=1}^{n} \beta_{i}\left(f_{i}^{\prime}(z) \mathrm{e}^{g_{i}(z)}\right)^{\beta_{i}-1}\left(f_{i}^{\prime \prime}(z) \mathrm{e}^{g_{i}(z)}+f_{i}^{\prime}(z) \mathrm{e}^{g_{i}(z)} g_{i}^{\prime}(z)\right) \prod_{\substack{k=1 \\ k \neq i}}^{n}\left(f_{k}^{\prime}(z) \mathrm{e}^{g_{k}(z)}\right)^{\beta_{k}} .
$$

From (29) and (30), we get: 


$$
\frac{G_{n}^{\prime \prime}(z)}{G_{n}^{\prime}(z)}=\sum_{i=1}^{n} \beta_{i}\left(\frac{f_{i}^{\prime \prime}(z)}{f_{i}^{\prime}(z)}+g_{i}^{\prime}(z)\right)
$$

Using relation (31) the function $p$ has the form:

$$
p(z)=\frac{1}{2\left|\beta_{1} \beta_{2} \cdots \beta_{n}\right|} \sum_{i=1}^{n} \beta_{i}\left(\frac{f_{i}^{\prime \prime}(z)}{f_{i}^{\prime}(z)}+g_{i}^{\prime}(z)\right) .
$$

We have:

$$
p(0)=\frac{\beta_{1}\left(2 a_{2}^{1}+1\right)+\beta_{2}\left(2 a_{2}^{2}+1\right)+\cdots+\beta_{n}\left(2 a_{2}^{n}+1\right)}{2\left|\beta_{1} \beta_{2} \cdots \beta_{n}\right|} .
$$

By using the relations (24), (25) and (28), we obtain:

$$
|p(z)| \leq \frac{1}{2\left|\beta_{1} \beta_{2} \cdots \beta_{n}\right|} \sum_{i=1}^{n}\left|\beta_{i}\left(\frac{f_{i}^{\prime \prime}(z)}{f_{i}^{\prime}(z)}+g_{i}^{\prime}(z)\right)\right| \leq \frac{1}{2\left|\beta_{1} \beta_{2} \cdots \beta_{n}\right|} 2 \sum_{i=1}^{n}\left|\beta_{i}\right| \leq 1
$$

and

$$
|p(0)|=\frac{\left|\beta_{1}\left(2 a_{2}^{1}+1\right)+\beta_{2}\left(2 a_{2}^{2}+1\right)+\cdots+\beta_{n}\left(2 a_{2}^{n}+1\right)\right|}{2\left|\beta_{1} \beta_{2} \cdots \beta_{n}\right|}=|c| .
$$

Applying Remark 1.3 for the function $p$, we obtain:

$$
|p(z)|=\frac{1}{2\left|\beta_{1} \beta_{2} \cdots \beta_{n}\right|}\left|\frac{G^{\prime \prime}(z)}{G^{\prime}(z)}\right| \leq \frac{|z|+|p(0)|}{1+|p(0)||z|} \leq \frac{|z|+|c|}{1+|c||z|} .
$$

From (34), we get:

$$
\left|\left(1-|z|^{2}\right) z \frac{G_{n}^{\prime \prime}(z)}{G_{n}^{\prime}(z)}\right| \leq\left|\beta_{1} \beta_{2} \cdots \beta_{n}\right| 2\left(1-|z|^{2}\right)|z| \frac{|z|+|c|}{1+|c||z|},
$$

for all $z \in U$.

Let us consider the function $Q:[0,1] \rightarrow R$

$$
Q(x)=2\left(1-x^{2}\right) x \frac{x+|c|}{1+|c| x}, x=|z| .
$$

Since $Q\left(\frac{1}{2}\right)=\frac{3}{4} \frac{1+2|c|}{2+|c|}>0$, it results:

$$
\max _{x \in[0,1]} Q(x)>0 .
$$

Using this result and the form (35), we have:

$$
\left|\left(1-|z|^{2}\right) z \frac{G_{n}^{\prime \prime}(z)}{G_{n}^{\prime}(z)}\right| \leq\left|\prod_{i=1}^{n} \beta_{i}\right| \max _{|z|<1}\left[2\left(1-|z|^{2}\right)|z| \frac{|z|+|c|}{1+|c||z|}\right],
$$

for all $z \in U$.

Applying the condition (26) in relation (36), we obtain:

$$
\left(1-|z|^{2}\right)\left|\frac{z F_{n}^{\prime \prime}(z)}{F_{n}^{\prime}(z)}\right| \leq 1,
$$

for all $z \in U$ and from Theorem 1.1, we have $G_{n} \in S$. 
Corollary 2.7. Let $\beta$ be a complex number and the functions $f \in S, f(z)=z+a_{2} z^{2}+\cdots, g \in S$, $g(z)=z+b_{2} z^{2}+\cdots$. If

$$
\left|\frac{f^{\prime \prime}(z)}{f^{\prime}(z)}\right|<1 \text { and }\left|g^{\prime}(z)\right|<1
$$

for all $z \in U$ and the constant $|\beta|$ satisfies the condition:

$$
|\beta| \leq \frac{1}{\max _{|z| \leq 1}\left[2|z|\left(1-|z|^{2}\right) \frac{2|z|+\left|2 a_{2}+1\right|}{2+\left|2 a_{2}+1\right||z|}\right]},
$$

then the function

$$
G_{1}(z)=\int_{0}^{z}\left(f^{\prime}(t) \mathrm{e}^{g(t)}\right)^{\beta} \mathrm{d} t
$$

is in the class $S$.

Proof. We consider $n=1$ in Theorem 2.6.

Remark 2.8. For $n=1, \mathrm{e}^{g_{1}(t)}=1, \quad \beta_{1}=\beta, f_{1}=f \quad$ in relation (27), we obtain the integral operator $G_{\beta}(z)=\int_{0}^{z}\left(f^{\prime}(t)\right)^{\beta} \mathrm{d} t$, defined and studied by V. Pescar in [8] [9].

Remark 2.9. For $n=1$ and $\beta=\alpha$ in relation (27), we obtain the integral operator $I_{1}(f, g)(z)=\int_{0}^{z}\left(f^{\prime}(t) \mathrm{e}^{g(t)}\right)^{\alpha} \mathrm{d} t$, introduced and studied by N. Ularu and D. Breaz in [10] and [11].

\section{Acknowledgements}

This work was supported by the strategic project PERFORM, POSDRU 159/1.5/S/138963, inside POSDRU Romania 2014, co-financed by the European Social Fund-Investing in People.

\section{References}

[1] Becker, J. (1972) Lownersche Differentialgleichung und quasikonform fortsetzbare schlichte Funktionen. Journal für die Reine und Angewandte Mathematik, 255, 23-43.

[2] Nehari, Z. (1952) Conformal Mapping. McGraw-Hill Book Company, New York.

[3] Alexander, J.W. (1915) Functions Which Map the Interior of the Unit Circle upon Simple Regions. Annals of Mathematics, 17, 12-22. http://dx.doi.org/10.2307/2007212

[4] Pescar, V. (1997) On Some Integral Operations Which Preserve the Univalence. Journal of Mathematics, 30, 1-10.

[5] Pescar, V. (1998) On the Univalence of an Integral Operator. Studia Universitatis “Babeș-Bolyai”, Cluj-Napoca, Mathematica, 43, 95-97.

[6] Breaz, D. and Breaz, N. (2002) Two Integral Operators. Studia Universitatis “Babeş-Bolyai”, Cluj-Napoca, Mathematica, 3, 13-21.

[7] Breaz, D. (2008) Certain Integral Operators on the Classes $\mathrm{M}\left(\beta_{i}\right)$ and $\mathrm{N}\left(\beta_{i}\right)$. Journal of Inequalities and Applications, Article ID: 719354.

[8] Pescar, V. (1997) Some Integral Operators and Their Univalence. The Journal of Analysis, 5, 157-162.

[9] Pescar, V. (1997) An Integral Operator Which Preserves the Univalency. The Annual Conference of the Romanian Society of Mathematical Sciences, Bucharest, 29 May-1 June 1997, 179-181.

[10] Ularu, N. and Breaz, D. (2012) Univalence Criterion and Convexity for an Integral Operator. Applied Mathematics Letter, 25, 658-661. http://dx.doi.org/10.1016/j.aml.2011.10.011

[11] Ularu, N. and Breaz, D. (2013) Univalence Condition and Properties for Two Integral Operators. Applied Sciences, 15, 112-117. 
Scientific Research Publishing (SCIRP) is one of the largest Open Access journal publishers. It is currently publishing more than 200 open access, online, peer-reviewed journals covering a wide range of academic disciplines. SCIRP serves the worldwide academic communities and contributes to the progress and application of science with its publication.

Other selected journals from SCIRP are listed as below. Submit your manuscript to us via either submit@scirp.org or Online Submission Portal.
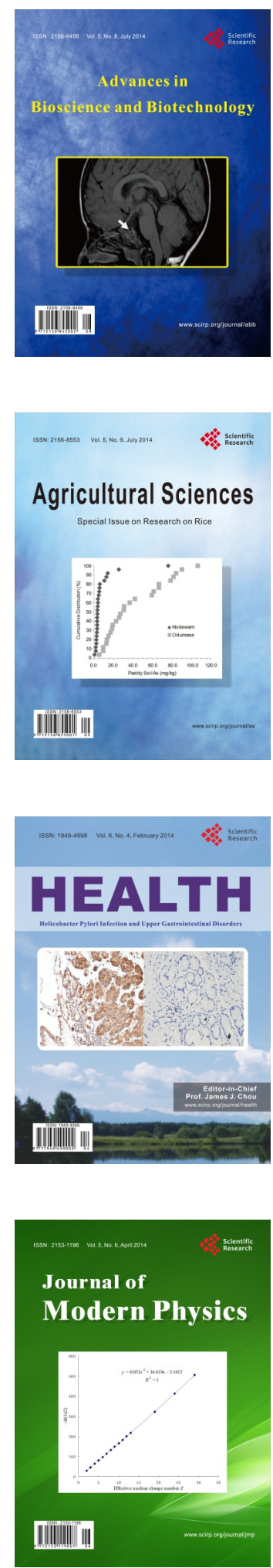
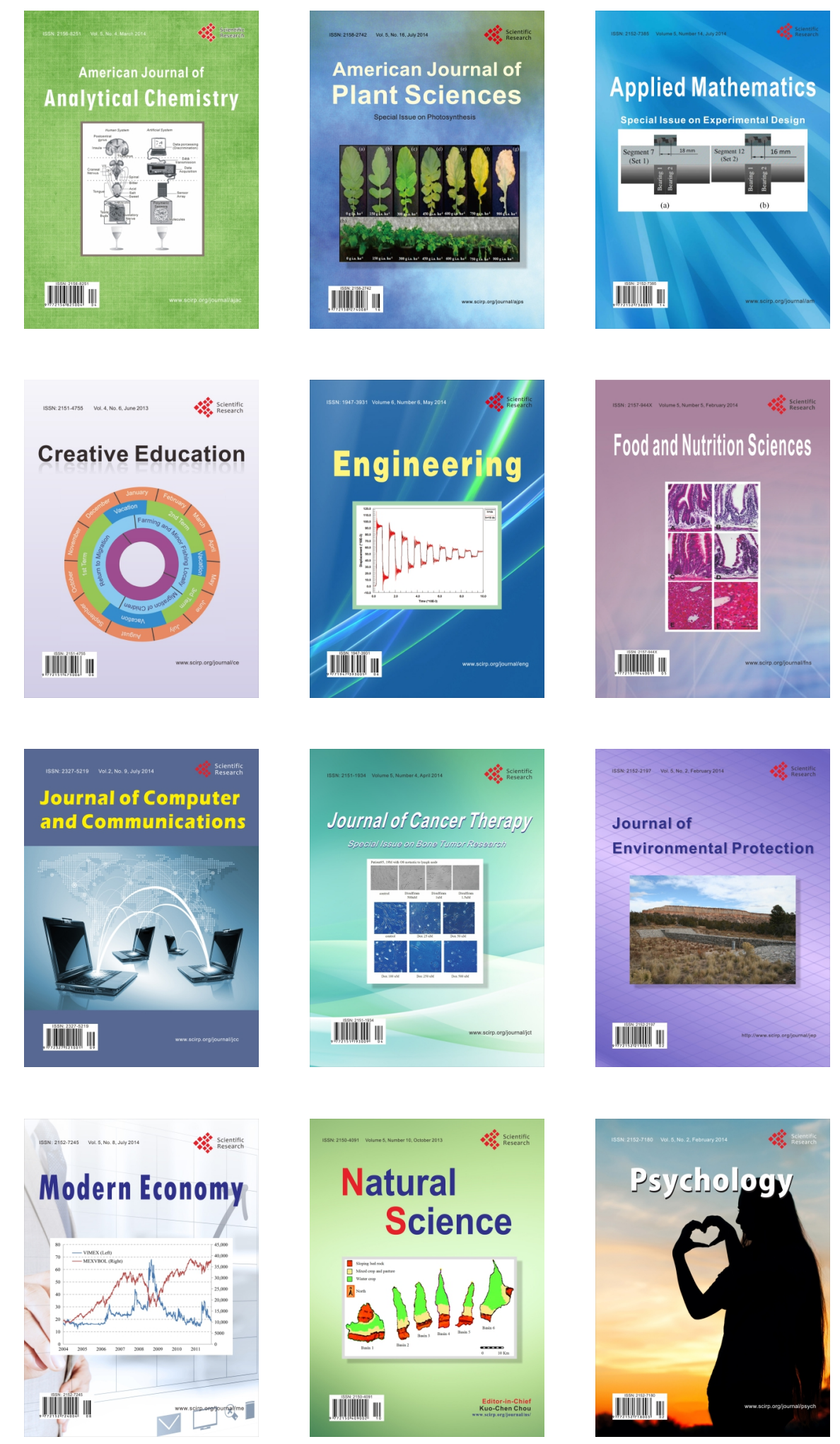